Lait 85 (2005) I-II

(C) INRA, EDP Sciences, 2005

DOI: $10.1051 /$ lait:2005030

\title{
Foreword
}

\section{2nd International Symposium on Spray Drying of Milk Products}

Teagasc, Dairy Products Research Centre (DPRC) was particularly pleased to welcome participants to the 2nd International Symposium on Spray Drying of Milk Products in Cork during 19th - 21st October 2004. The subject of spray drying continues to be of considerable importance to the dairy industry as it enables distant markets for dairy products to be reached via preserved milks. On-going innovation in the functionality of milk powders and adaptation of key process technologies is critical to the development of a new generation of health promoting food ingredients.

Many will recall the inaugural Symposium held in Rennes, France in 2001 which was inspired by J.-L. Maubois and P. Schuck, INRA Dairy Research Laboratory. We would like to acknowledge the continued association of INRA with this event, and in particular the assistance of Dr. Pierre Schuck with the organisation of the symposium. The original objectives were retained, i.e. to update a "progress report on current knowledge in the field of spray drying (...) to carry out scientific exchanges between specialists in the area, dairy companies and equipment manufacturers." In the meantime, the international scientific advisory committee was expanded to include additional milk drying technology experts from around the world, and we would like to acknowledge their support and assistance.

Following a positive response to the Call for Papers, volunteered oral contributions were distributed according to the selected themes and keynote addresses: (i) physical properties, (ii) processing, sensors and process control, (iii) quality, safety, nutrition and (iv) dairy powders and applications. Dairy Powders and Applications on day 3 incorporated the Fifth Food Ingredients Symposium - a continuation of the successful series of DPRC symposia due to be held. Since dried dairy ingredients are allied closely to the spray drying of milk products, it was considered appropriate to integrate both programmes on this occasion.

This 2nd International Symposium also presented DPRC with an opportunity to inform the audience of its dissemination role in the EU-funded thematic network SPRAYNET Technology Transfer of Application Protocols, Standards and Health/Safety Criteria for the use of Spray Drying Technology (www.spray-net.com). Both oral presentations and posters were presented by several SPRAYNET partners throughout the 3-day event. 
In keeping with previous practice, we are pleased that EDP Sciences have continued their relationship with the symposium series by affording speakers the opportunity to have their papers refereed and published in a proceedings special issue of Le Lait - Dairy Science and Technology journal. Dr. Donal O'Callaghan coordinated the management of the scientific editing process.

Finally, I would like to pay tribute to my colleague, Dr. Kieran Keogh for his time and commitment to the organisation of the symposium programme, and coordination of the Organising Committee. The Symposium marked the end of a fruitful career as a senior researcher at DPRC, following which his planned retirement took effect.

The 3rd International Symposium in the series will be hosted by Professor Phil Tong, Dairy Products Technology Center, California Polytechnic State University (Calpoly), San Louis Obispo, CA in 2007.

Philip M. Kelly

Teagasc-DPRC 